\title{
Spectral Properties of 3-Benzanthrone Derivative Dyes in Isotropic Solvents, Polymer Film and Liquid Crystal
}

\author{
I. Grabchev, I. Moneva, E. Wolarz ${ }^{\mathrm{a}}$, D. Bauman ${ }^{\mathrm{a}}$, and S. Stoyanov ${ }^{\mathrm{b}}$ \\ Institute of Polymers, Bulgarian Academy of Sciences, Sofia 1113, Bulgaria \\ ${ }^{a}$ Faculty of Technical Physics, Poznan University of Technology, Poznan, Poland \\ ${ }^{\mathrm{b}}$ Faculty of Chemistry, University of Sofia, Sofia, Bulgaria \\ Reprint requests to Dr. I. G.; Fax: +359(2)70-75-23; E-mail: grabchev@polymer.bas.bg
}

Z. Naturforsch. 56a, 291-296 (2001); received January 24, 2001

Some recently synthesized benzanthrone derivatives bearing azomethine and oxy groups at C-3 position have been systematically studied. The influence of the substituents and of the environment (isotropic and anisotropic media) on the absorption and fluorescence of the dyes and their vibronic transitions is discussed in this paper.

Key words: 3-Benzanthrone Derivatives; Azomethine and Oxysubstitutes; Absorption; Fluorescence; Vibronic Structure.

\section{Introduction}

Derivatives of 3-amino- and 3-oxybenzanthrone exhibit, both in solution and in the solid state, bright yellow-green or orange-red fluorescence depending on their chemical structure [1]. Because of their excellent colour characteristics and high photostability, the dyes are of wide practical interest for a variety of applications as daylight fluorescent pigments [2], disperse dyes for polyester fibres [3], for chemical coloration and photostabilization of polymeric materials [4], and also as laser dyes [5].

Recently, it has been shown that benzanthrone dyes can also be appropriate components in "guest-host" liquid-crystal (LC) systems for electro-optic displays $[6,7]$. In a "guest-host" LC display, a dichroic dye (guest) is dissolved in a nematic liquid crystalline host which acts as anisotropic matrix orienting the dye molecules under electric voltage, thus causing selective absorption by the dyes [8].

In this paper we report the spectral properties of recently synthesized 3-azomethine and 3-oxy benzanthrone derivatives measured in isotropic and anisotropic media, with accent on their potential use in "guesthost" systems for LC displays. Essentially, the influence of the substituents and of the media including LC and polymer films upon the absorption and fluorescence properties of the dyes and their vibronic transitions has been studied.

\section{Experimental}

The dyes under study have the following general formula:

$$
\begin{aligned}
& \mathrm{R}= \\
& -\mathrm{NH} \\
& -\mathrm{N}=\mathrm{CH}-\mathrm{C}_{6} \mathrm{H}_{5} \\
& -\mathrm{N}=\mathrm{CH}-\mathrm{C}_{6} \mathrm{H}_{4} \mathrm{OH}-\mathrm{O} \\
& -\mathrm{N}=\mathrm{CH}-\mathrm{C}_{6} \mathrm{H}_{4} \mathrm{OH}-\mathrm{p} \\
& -\mathrm{N}=\mathrm{CH}-\mathrm{C}_{6} \mathrm{H}_{4} \mathrm{~N}\left(\mathrm{CH}_{3}\right)_{2}-\mathrm{p} \\
& -\mathrm{OCH}_{3} \\
& -\mathrm{OCH}_{2} \mathrm{CH}_{2} \mathrm{OCH}_{3} \\
& -\mathrm{OC}_{6} \mathrm{H}_{5} .
\end{aligned}
$$

Their synthesis has recently been described $[7,9]$.

The electronic spectra of the dyes in various media including isotropic solvents, LC and polystyrene (PS) films were recorded on a Specord M42 spectrometer with $2 \mathrm{~nm}$ resolution at room temperature. For recording the polarised absorption spectra, UV neutral filters were used. The dye concentration in isotropic solutions was $10^{-5} \mathrm{Mol} \mathrm{1}^{-1}$.

The fluorescence spectra of the dye/LC systems were measured by means of a home-made fluorimeter in $\pi$ geometry, i.e. the exciting light beam was perpendicular to the cell surface and the emitted fluorescence light was registered perpendicular to the surface, from the same side of the cell. The wavelength of the exciting radiation corresponds to the absorption maxima of the dyes. The quantum fluorescence yield $\Phi_{\mathrm{F}}$ was determined on the basis of absorption and fluorescence spectra of the dyes in ethanol at a concentration of $1.10^{-1} \mathrm{Mol} \mathrm{l}^{-1}$. Rhodamine $6 \mathrm{G}\left(\Phi_{\mathrm{O}}=0.88\right)$ was used as a standard [10]. 
Commercial LC ZLI 1840 (Merck, Germany) was used as a host. It exhibits a stable nematic phase over a broad temperature range $\left(-15^{\circ} \div 90^{\circ} \mathrm{C}\right)$ and high positive dielectric anisotropy [11]. The dyes were initially screened for solubility in LC and then dissolved in the medium at a concentration of $0.5 \mathrm{w} / \mathrm{v} \%$, which is suitable for the intended application [12]. The measurements on the dyes in LC were carried out in "sandwich" cells of $20 \mu \mathrm{m}$ thickness. Planar homogeneous molecular orientation of the dye/LC systems in the cells was achieved by coating the cell surfaces from inside with polyimide layers orientated by rubbing $[13,14]$.

Coloured thin films of polystyrene $(0.5 \%$ dye) were prepared from a $10 \%$ solution of polystyrene in benzene with the dye dissolved there.

\section{Results and Discussion}

\section{Influence of the Substituents at C-3 Position on the Dyes Absorption}

The electron donor-acceptor interaction in the molecule of 3-substituted benzanthrone occurs between the electron acceptor carbonyl group and the electron donating groups at C-3 position of the chromophoric system [9]

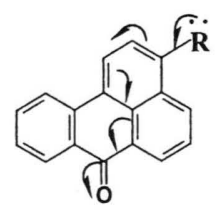

Scheme 1

The molecules are polarised in dependence on the electron donating power of the substituent $\mathrm{R}$. Therefore their absorption properties are different.

The 3-substituted benzanthrone derivatives exhibit the following order of the energetic levels $[15,16]$

$$
\mathrm{T} \pi \pi^{*}<\mathrm{S} \pi \pi^{*}<\mathrm{Tn} \pi^{*}<\mathrm{Sn} \pi^{*} .
$$

In the absorption spectra of these compounds, the longwave band in the visible region has charge transfer, CT, character, due to $\pi \rightarrow \pi^{*}$ electron transfer during the $\mathrm{S}_{0} \rightarrow \mathrm{S}_{1}$ transition. The absorption and emission bands largely depend both on the electron donating power of the substituents at C-3 position and the nature of solvents. The absorption properties of the dyes determined in a polar proton donating solvent (ethanol) and in a nonpolar solvent (benzene) are presented in Table 1. The exchange of the polar solvent with the nonpolar one
Table 1. Absorption maxima $\lambda_{\mathrm{A}}$ of substituted 3-benzanthrone dyes in ethanol, benzene and polystyrene (PS) and other photophysical characteristics of the dyes determined in ethanol (see text).

\begin{tabular}{lcccccccc}
\hline Dyes & 1 & 2 & 3 & 4 & 5 & 6 & 7 & 8 \\
\hline$\lambda_{\mathrm{A}} \mathrm{nm}$ & & & & & & & & \\
$\quad$ in ethanol & 508 & 430 & 428 & 516 & 470 & 430 & 436 & 432 \\
$\quad$ in benzene & 465 & 426 & 421 & 464 & 459 & 419 & 417 & 418 \\
$\quad$ in PS & 464 & 424 & 420 & 426 & 460 & 422 & 418 & 420 \\
$\log \varepsilon$ & 3.96 & 4.20 & 4.23 & 4.16 & 4.12 & 4.01 & 4.02 & 4.04 \\
$\lambda_{\mathrm{F}} \mathrm{nm}$ & 568 & 525 & 569 & 653 & 594 & 525 & 526 & 532 \\
$\left(v_{\mathrm{A}}-v_{\mathrm{F}}\right) \mathrm{cm}^{-1}$ & 2079 & 4208 & 5789 & 4066 & 4441 & 4208 & 3924 & 4351 \\
$E_{\mathrm{S} 1 \mathrm{kJmol}^{-1}}$ & 224.1 & 253.6 & 252.5 & 192.7 & 211.5 & 246.8 & 247.3 & 245.3 \\
$F$ & 0.181 & 0.279 & 0.326 & 0.293 & 0.281 & 0.186 & 0.186 & 0.188 \\
$\Phi_{\mathrm{F}}$ & 0.006 & 0.008 & 0.02 & - & - & 0.55 & 0.49 & 0.42 \\
\hline
\end{tabular}

brings in most cases a substantial hypsochromic shift, which is well known for CT bands [17].

Let us consider the influence of both groups of substituents, azomethine and oxy groups, upon the absorption properties of the dyes. In the case of dye 1, the CT band exhibits a bathochromic effect because of the strong donor-acceptor interaction. The substitution of the primary amino group with the azomethine group (dyes 2-5) leads to decrease of the donor-acceptor interaction and, correspondingly, to hypsochromic shift of the absorption maximum, with the exception of dye 4 . The different nature and position of the substituents in the benzene ring of the azomethine fragment in dyes 2-5 cause also themselves a substantial effect upon the electronic spectra. Dye 2, with no substituents in the benzene ring, exhibits a yellow-green colour in ethanol and benzene solutions. In the case of dye 3 , the electron donating $\mathrm{OH}$ - group at o-position with regard to the azomethine group changes only weakly the position of the CT band compared to that of dye 2. Most probably this can be related to the formation of an intramolecular hydrogen bond according to Scheme 2:<smiles>O=C1c2ccccc2-c2ccc(N=Cc3ccccc3O)c3cccc1c23</smiles>

Scheme 2

The electron donating $\mathrm{OH}$ and $-\mathrm{N}\left(\mathrm{CH}_{3}\right)_{2}$ groups at p-position in the benzene ring (dyes 4 and 5) cause stronger 
polarisation of the benzanthrone molecule due to enhanced donor-acceptor interaction. Consequently, their absorption maxima are bathochromically shifted with regard to those of dye 2 .

The substitution of the primary amino group in dye 1 with alkoxy and phenoxy groups (dye 6-8) leads to even larger hypsochromic shifts of the absorption maxima than for dyes 3-5. This is related to the weaker electron donating nature of the OR groups in comparison with the primary amino group. In the case of the oxy derivative dyes $6-8$, there is no substantial influence of the substituents connected to the $\mathrm{O}$ atom at $3^{\text {rd }}$ position on the positions of the CT bands.

\section{Influence of the Environment on Dyes Absorption}

The spectral properties of benzanthrone dyes depend also on the nature of the environment (polarity, viscosity, formation of hydrogen bonds or other intermolecular interactions, etc.) [18-20]. The dependence of the absorption maximum of the CT bands in the case of dyes 1,2 and 6, as example, upon the empirical parameter of solvent polarity $\mathrm{E}_{\mathrm{T}}(30)$ [21] is presented in Figure 1. It is seen from Fig. 1 that the dyes 1, 2 and 6 exhibit positive solvatochromism. The linear relations observed indicate that dipol-dipol interactions prevail in the dye solution. In acetone, a specific interaction between the free electron pair of the $\mathrm{O}$ atom of the solvent and $\mathrm{H}$ atoms of the primary amino group in dye 1 is possible. In this case, the polarisation of the molecule is enhanced and the absorption maxima are bathochromically shifted with respect to those in toluene, benzene and chloroform, where such intermolecular H-bond complex formation cannot occur. The absorption maxima of dye 1 in the proton donating solvents ethanol and methanol are bathochromically shifted to 508 and $512 \mathrm{~nm}$, respectively. In that case, the polarisation of the chromophoric system is enhanced by the formation of intermolecular $\mathrm{H}$-bonds between the free electron pair of the carbonyl group in benzanthrone and the hydroxy $\mathrm{H}$-atoms of the solvents, which causes the bathochromic shift. Dyes 2 and 6 have similar absorption behaviour in organic solvents. For both dyes the absorption maxima in alcoholic solvents are bathochromically shifted in comparison with those in non polar aprotic solvents. From Fig. 1 it is seen too that the absorption maxima of dyes 2 and 6 are less influenced by the nature of the solvents.

All benzanthrone dyes under study have similar absorption properties in proton donating solvents and, correspondingly, their long-wave CT bands are strongly

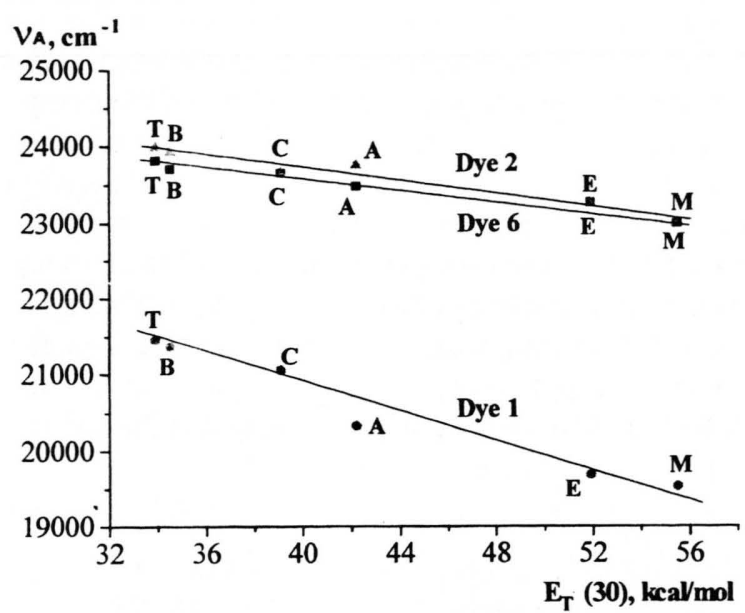

Fig. 1. Dependence of absorption frequencies of benzanthrone dyes in different media on the empirical solvent polarity parameter $E_{\mathrm{T}}(30)$. The media: T: toluene, B: benzene, $\mathrm{C}$ : chloroform, A: acetone, E: ethanol, and M: methanol.

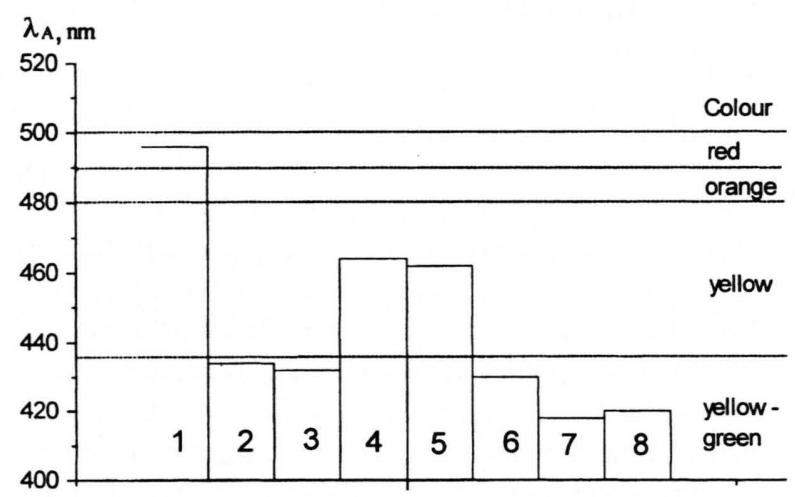

Fig. 2. Absorption maxima of the 3-benzanthrone derivative dyes $1-8$ in LC.

bathochromically shifted with regard to those in benzene and toluene. In acetone, intermolecular hydrogen bonds are formed only in case of the dyes 1 and 4, by the labile $\mathrm{H}$ atoms of amino and hydroxy groups.

The absorption properties of dyes 1-8 in solid polystyrene (PS) film (Table 1) are similar to those in nonpolar solvents as a result of comparable polarity constants: $\varepsilon$ (benzene) $=2.28, \varepsilon(\mathrm{PS})=2.40-2.65$ [22]. An exception is dye 4 where the absorption maximum in PS is largely hypsochromically shifted $(\Delta \lambda=34 \mathrm{~nm})$ with regard to that in benzene, probably because of aggregate formation and lack of conformational changes of the dye in the solid matrix. 
Figure 2 presents the maxima of the CT band of the dyes in LC. As shown, dyes 1-8 cover the colour interval from yellow-green to red depending on their structural specificity. Dye 1 has an intense red colour while dyes $2,3,6-8$ have yellow-green colour, enhanced in the case of dyes $6-8$ by their strong green fluorescence; dyes 4 and 5 exhibit yellow colour. The absorption and fluorescence maxima of the dyes in LC are bathochromically shifted with regard to $\lambda_{\mathrm{A}}$ and $\lambda_{\mathrm{F}}$ in the nonpolar solvents in polystyrene film, and hypsochromically shifted in the polar solvents, indicating that the polarity of LC is in-between.

\section{Fluorescence and other Optical Characteristics of the Dyes in Isotropic and Anisotropic Media}

It is known that the substituents at C-3 position of the benzanthrone molecule have strong influence on all the basic photophysical characteristics [18, 19, 23]. This is established also here not only for the absorption maxima but also for the other characteristics of the dyes under study (Table 1).

The fluorescence maxima of these dyes are in the region $525-653 \mathrm{~nm}$, depending on the structure of the chromophore. In the series of $\mathrm{N}$-substituted derivatives (dyes 1-5), the nature of the substituent has a strong influence on the fluorescence maxima $\lambda_{\mathrm{F}}$. The substitution of the primary amino group in dye 1 by the arylazomethine group (dye 2) leads to a large blue shift of the fluorescence maximum, of about $40 \mathrm{~nm}$. Substantial influence on the fluorescence maxima show also the substituents in the azomethine group. The electron donating dimethylamino group in the benzene ring in p-position (dye 5) causes a large red shift of about $70 \mathrm{~nm}$. The hydroxy group in o-position (dye 3 ) causes also a red shift of the fluorescence maximum due to the intramolecular hydrogen bond formation (Scheme 2), suggesting that the hydrogen bond is more stable in the excited $S_{1}$ state than in the ground state $S_{0}$. The largest red shift of the fluorescence maximum $\lambda_{\mathrm{F}}$, of $128 \mathrm{~nm}$ (dye 4 with hydroxy group in p-position) is probably connected with the prototropic state of the dye molecule presented in Scheme 3 [9]:

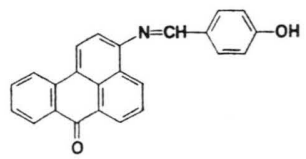

(4)

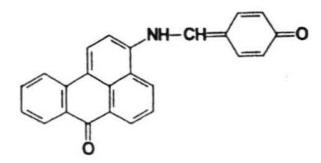

(4')
Scheme 3
Table 2. Optical characteristics of substituted 3-benzanthrone dyes in liquid crystal $(1 / d$-molecular aspect ratio, $D(\lambda)$ : linear dichroism measured by absorption $A$ or fluorescence $F$ ).

\begin{tabular}{lcccccccc}
\hline Dyes & 1 & 2 & 3 & 4 & 5 & 6 & 7 & 8 \\
$1 / d$ & 1.10 & 1.17 & 1.17 & 2.18 & 2.43 & 1.31 & 2.02 & 1.53 \\
\hline$\lambda_{\mathrm{A}} \mathrm{nm}$ & 496 & 434 & 432 & 464 & 462 & 430 & 418 & 420 \\
$D\left(\lambda_{\mathrm{A}}\right)$ & 2.76 & 4.54 & 5.03 & 4.65 & 4.62 & 3.64 & 3.04 & 3.76 \\
$\lambda_{\mathrm{F}} \mathrm{nm}$ & - & - & - & - & - & 502 & 504 & 502 \\
$D\left(\lambda_{\mathrm{F}}\right)$ & - & - & - & - & - & 1.84 & 2.06 & 2.82 \\
\hline
\end{tabular}

In the case of alkoxy and phenoxy substituted benzanthrone dyes, the substituents have almost no influence on the fluorescence maxima.

The energy of the first excited state $E_{\mathrm{S} 1}$ of dyes 1-8 in ethanol (Table 1) is determined from the cross point of the absorption and fluorescence spectra. Dyes 4 and 5 show the lowest values, i.e. they need lower energy supply for the $S_{0} \rightarrow S_{1}$ transition.

The substituents at C-3 have definitely a strong influence on the quantum yields of fluorescence $\Phi_{\mathrm{F}}$ (Table 1). In the case of the $\mathrm{N}$-substituted derivatives, the quantum yield of fluorescence is very low, indicating a substantial nonradiating deactivation during the transition from the excited to the ground state. Much higher values of the quantum yield have the oxy substituted benzanthrone dyes, where $\Phi_{\mathrm{F}}=0.42-0.55$, their molecules being subjected to less conformational changes.

An important characteristic of the dyes is the oscillator strength $f$ determined by [24]

$$
f=4.32 \times 10^{-9} \Delta v_{1 / 2} \varepsilon_{\max },
$$

where $\Delta v_{1 / 2}$ is the width of the absorption band (in $\mathrm{cm}^{-1}$ ) at $1 / 2 \quad \varepsilon_{\max }$. The $f$ values are highest for arylazomethine derivatives (dyes 2-5), showing the tendency to increase with the higher molar absorption (Table 1).

For the potential use of the dyes in the "guest-host" LC displays it is of interest to evaluate the fluorescence properties of dyes 1-8 in LC matrix. In the case of dyes $1-5$, however, one could observe on irradiation with UV-light only a faint fluorescence emission of orangered colour. In the case of dyes 6-8, bearing oxy groups, the emission is very intensive in the range $450-650 \mathrm{~nm}$, where the maximum of fluorescence is observed at 502-504 nm (Table 2).

It is known that some dyes, when oriented selectively to absorb polarised light, exhibit dichroism [25]. Figure 3 presents as an example the polarised absorption spectra of dye 3 in LC. From such spectra, the dichroic 


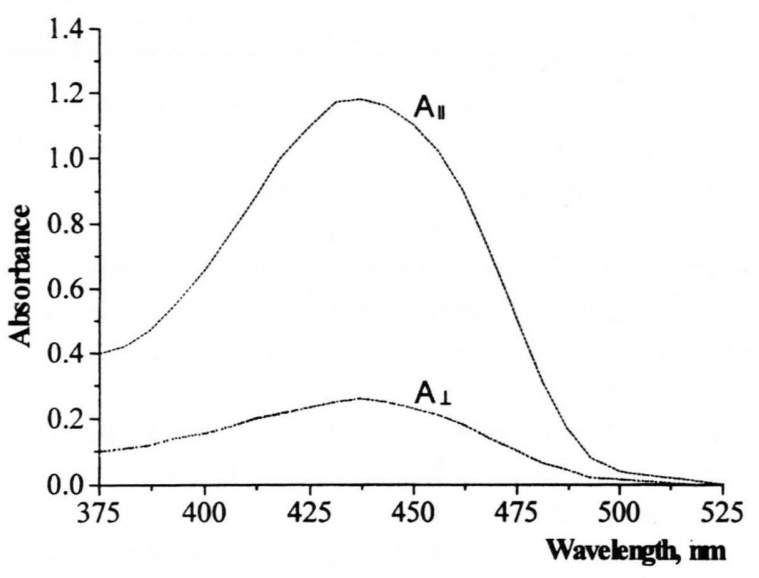

Fig. 3. Polarised absorption spectra of dye 3 in LC. $A_{\|}$and $A_{\perp}$ are the absorbancies in polarised light at parallel and perpendicular orientation of the polarizer with respect to the LC director, respectively.

ratio of the dyes in $\mathrm{LC}$ is determined according to the equation [26]

$$
D\left(\lambda_{\mathrm{A}}\right)=A_{\|} / A_{\perp},
$$

where $\lambda_{\mathrm{A}}$ is the absorption maximum, and $A_{\|}$and $A_{\perp}$ are the corresponding absorbances in polarised light at parallel and perpendicular orientation of the polarizer with respect to the LC director, respectively. The data obtained (Table 2) show that the dichroic ratios of the benzanthrone dyes under study are not proportional to the length to width ratio, $1 / d$, of the molecules and obviously depend not only on the linearity of the molecules but also on other factors. The calculation of $1 / d$ values was made by using the bond lengths in the molecules including the end groups [27].

It is evident that both substituent $\mathrm{R}$ and the flexibility of the C-N-C link at C-3 of the benzanthrone molecule influence the dichroic properties of dyes 1-8. Azomethine derivatives (dyes 2-5) show higher values of $D\left(\lambda_{\mathrm{A}}\right)$ than the oxy derivatives $6-8$, suggesting better orientational order of their solutions in LC [28]. The azomethine fragment there is involved in a system of conjugated double bonds of the chromophoric system, in this way increasing its linearity, while the oxy substituents at C-3 of dyes 6-8 exhibit only their electron donating power. In Table 2 , the respective values of the dichroic ratio determined from the fluorescence maxima, $D\left(\lambda_{\mathrm{F}}\right)$, are also presented. The values are lower than those determined from the absorption maxima, $D\left(\lambda_{\mathrm{A}}\right)$, which is probably due to the existence of non zero intra-
Table 3. Vibronic modes of substituted 3-benzanthrone dyes in the visible region (in $\mathrm{cm}^{-1}$ ).

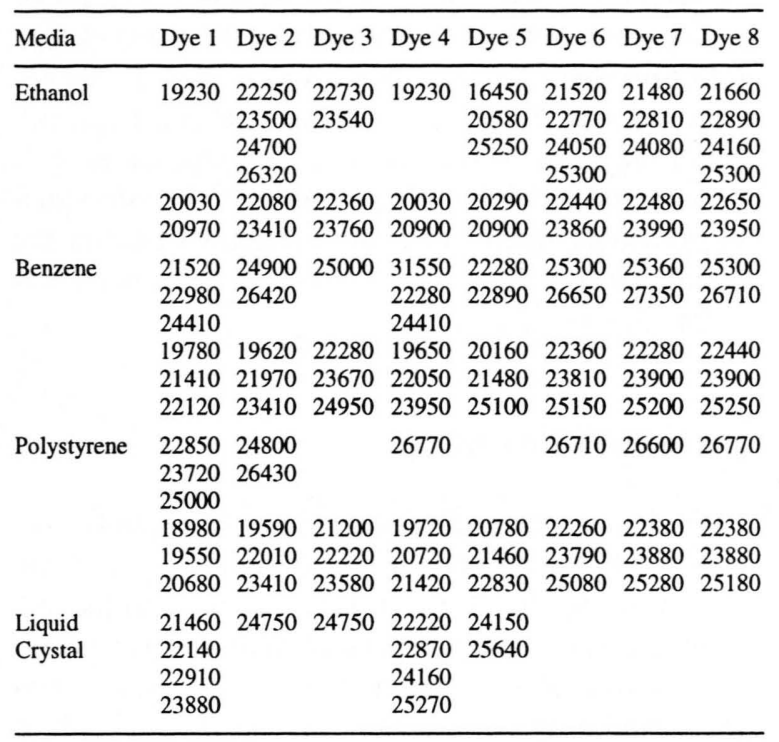

molecular angles between the absorption and emission oscillators $[29,30]$.

\section{Vibronic Transitions in Benzanthrone Dyes}

The width of an absorption band in the electronic spectra of conjugated substances is mainly a result of both vibronic and intermolecular interactions.

The observed vibronic modes of all benzanthrone dyes studied in different media, ethanol, benzene, PS film and in LC cell, are determined from the dyes second derivative spectra [31] and are presented in Table 3. Vibronic transitions within the $S_{0} \rightarrow S_{1}$ transition are less excited in the polar proton donating ethanol. When comparing the vibronic structure of azomethine and oxy derivative dyes, it is seen that the oxy substituted benzanthrone dyes (6-8) are characterised by a more pronounced vibronic structure of the spectra, probably connected with the greater rigidity of their molecules. This is also valid for the azomethine dye 2 .

The vibronic structure is more pronounced in benzene due to the lower solvent polarity and to the lack of hydrogen bond formation between dye and solvent. It is noteworthy that the vibronic structure of the dyes in benzene and PS film is similar, indicating that it depends substantially on the polarity of the medium but not on its viscosity. 
For the dyes oriented in LC, the vibronic structure of dyes 1-5 (especially of dye 1 and 4 ) is richer than that of the oxy derivatives 6-8. Obviously, the oriented state of LC prevents the possible conformational changes connected with a rotation of the azomethine fragment. Within the series of azomethine derivative dyes $2-5$, dyes 2 and 3 have the less pronounced vibronic structure, probably due to molecular rigidity which in the case of dye 3 is due to the intramolecular $\mathrm{H}$ bond formation (Scheme 2).

\section{Concluding Remarks}

The spectral properties of a number of recently synthesised 3-benzanthrone derivatives bearing azomethine and oxy groups have been determined in isotropic and anisotropic (LC) media. The substituents at 3-C position of the benzanthrone molecule have strong influence on the spectral properties of the dyes under study, which confirms the prevailing role of the electron donor-acceptor interaction. The influence is stronger in case of azomethine substitutes (dyes 2-5), where the polariza-

[1] B. Krassovitski and B. Bolotin, Organic Luminophores, L. Chimia, 1984 (in Russian).

[2] F. Carlini, C. Paffoni, and G. Boffa, Dyes and Pigments 3, 59 (1982)

[3] N. Ayangar, R. Lahoti, and R. Wagle, Indian J. Chem. 16B, 106 (1978).

[4] V. Bojinov and T. Konstantinova, Polym. Degr. Stab. 68, 295 (2000).

[5] N. Borisevich, V. Grusinskii, I. Kalocha, and B. Tolkachev, J. Appl. Spectr. 11, 173 (1969).

[6] I. Grabchev and I. Moneva, C. R. Bulg. Acad. Sci. 59, (1997).

[7] I. Grabchev and I. Moneva, Dyes and Pigments 37, 155 (1998).

[8] G. Heilmeier and L. Zanoni, Appl. Phys. Lett. 19, 91 (1968).

[9] I. Grabchev, V. Bojinov, and I. Moneva, J. Mol. Str. 471, 19 (1998)

[10] I. Olmsted, J. Phys. Chem. 83, 2581 (1979).

[11] Merck prospect: Liquid Crystal Mixtures for Electro-Optic Displays, Oct. 1994, Darmstadt (Germany).

[12] G. W. Gray, Chimia 34, 51 (1980)

[13] E. Wolarz, H. Morison, and D. Bauman, Dysplays 13, 171 (1992).

[14] I. Grabchev, I. Moneva, E. Wolarz, and D. Bauman, Z. Naturforsch. 51a, 1185 (1996).

[15] N. Proskuryakova and R. Nurmuknametov, Optics and Spectroscopy 27, 119 (1969)

[16] V. Bhujle and M. Radhye, Indian J. Chem. 9, 1405 (1971). tion is large, and smaller for the oxy derivatives with their lower polarization (dyes 6-8).

There is a well pronounced solvatochromic effect established for dyes having groups capable to solute-solvent interaction (dyes 1, 4 and 5). When such groups are not present (dyes 2, 6-8) or are screened (dye 3) or undergo transformation (dye 4), the effect is small.

The vibronic interactions of the dyes depend both on the nature of the substituents and the polarity of environment in a complex way. The dye molecules are more stable in the excited state $S_{1}$ in the nonpolar media benzene and polystyrene film and in the oriented state in the liquid crystal matrix.

The functional properties of the dyes for use in electro-optic displays can be resumed as follows. The dyes cover a wide colour interval, from yellow-green to redorange. The oxy substituted benzanthrone dyes are expected to enhance the image contrast of the displays because of their good fluorescence properties. The azomethine substituted dyes are also of interest because of their dichroic properties which suggest a better orientation order of the dye/LC system in the display and a well developed vibronic structure supposing enhanced contrast.

[17] R. Nurmuknametov, Absorption and Luminescence of Aromatic Compounds, M. Chimia, 1971 (in Russian).

[18] P. Bentley, J. McKeller, G. Phillips, and J. C. S. Perkin II, 523 (1974)

[19] P. Bentley, J. McKeller, and J. C. S. Perkin II, 1850 (1976).

[20] M. Nepras, O. Machalicky, M. Seps, R. Hrdina, P. Kapusta, and V. Fidler, Dyes and Pigments 35, 31 (1997).

[21] C. Reichard, Solvent Effects in Organic Chemistry, NY, Verlag Chemie, Gmbse 1979.

[22] Chemical Encyclopaedia, ed. I. Knunjanz, v.1, M., Soviet Encyclopaedia, 1961.

[23] N. Levchenko and L. Podgornaya, J. Appl. Spectr. 8, 164 (1968).

[24] P. Gordon and P. Gregory, Organic Chemistry in Colour, M. Chimia, 1987 (in Russian).

[25] A. Ivashchenko and V. Rumiantsev, Mol. Cryst. Liq. Cryst. 150, 1 (1987).

[26] A. Blinov, V. Kizel, and V. Rumiantsev, Krystalografiya 20, 1245 (1975).

[27] A. I. Kitaigorodskii, Organic Crystal Chemistry, M., Chimia, 1955 (in Russian).

[28] I. Grabchev, I. Moneva, E. Wolarz, and D. Bauman - in preparation.

[29] L. L. Chapoy and D. B. DuPre, J. Chem. Phys. 70, 2550 (1979).

[30] E. Wolarz, Z. Naturforsch. 47a, 807 (1992).

[31] N. Dimov, Organic Analysis, S., Technika (1984). 